\title{
When Stay-at-Home Orders Leave Victims Unsafe at Home: Exploring the Risk and Consequences of Intimate Partner Violence during the COVID-19 Pandemic
}

\section{Catherine Kaukinen ${ }^{1}$}

Received: 11 May 2020 / Accepted: 29 May 2020 /

Published online: 6 June 2020

(C) Southern Criminal Justice Association 2020

\begin{abstract}
The novel coronavirus pandemic (hereafter COVID-19) is likely to have unprecedented impacts on the incidence and impacts of crime and violence globally. This includes impacts to the risk, consequences, and decisionmaking of women experiencing violence by an intimate partner (hereafter IPV). Most importantly, the COVID-19 pandemic, and its impact on the risk of IPV is likely to differentially impact vulnerable populations, including minority women and those with long histories of victimization and mental health issues. This review paper explores the potential short- and long-term implications of COVID-19 on the risk of IPV, highlighting some of the most recent preliminary data. The economic impact of the COVID-19 pandemic, record levels of male unemployment, added stressors in the home, including the care and home schooling of children, and the social distancing measures required by the epidemiological response, may serve to undermine the decades of progress made in keeping women and children safe at home. Victim police reporting, help-seeking decisions, and social service utilization during the pandemic are likely to be impacted by stay-at-home orders and social distancing requirements. The paper concludes with a discussion of the implications for providing safety planning and self-care for victims and their children.
\end{abstract}

Keywords COVID-19 - Intimate partner violence $\cdot$ Social distancing and stay-at-home orders $\cdot$ Mental health and PTSD

Catherine Kaukinen

catherine.kaukinen@ucf.edu

1 Professor and Chair of Criminal Justice University of Central Florida, 12805 Pegasus Drive HPAI, Suite 311 Orlando, Florida 32816-1600 32816 - 2200, USA 


\section{Background}

The novel coronavirus pandemic (hereafter COVID-19) is likely to have unprecedented impacts on the incidence and impacts of crime and violence globally. This includes impacts to the risk, consequences, and decision-making of women experiencing violence by an intimate partner. There has been a dramatic decline in the risk of lethal and less-than-lethal intimate partner violence (hereafter IPV) since the early 1990s (Powers \& Kaukinen, 2012; Xie, Heimer, \& Lauritsen, 2012; Lauritsen \& Heimer, 2009). Catalano (2012) notes that between 1994 and 2010 there has been an overall decline of $64 \%$ in the rate of intimate partner violence in the United States. This decline can be found in both official data from law enforcement agencies, but also in survey-based victimization data. The decline in women's risk of lethal and less-than-lethal IPV is a function of women's declining risk exposure via changes in the economic status and well-being of women, declines in women's dependence on marriage (via increased employment of women, declining marriage rates, and increasing access to and rates of divorce), general awareness of the nature of intimate partner violence and decreasing stigma for victims to come forward and report that violence, and the expansion and availability of intimate partner violence services and interventions. The short- and longterm health consequences of COVID-19, stay-at-home orders and social distancing measures, family isolation, and the economic impacts of the pandemic are likely to impact both women's experience of IPV and their ability to navigate ending these violent relationships, and potentially reverse the declining trend in IPV. Boserup, McKenney, \& Elkbul (2020) note that the social isolation associated with quarantining and mandatory stay-at-home orders may worsen the economic and health vulnerabilities of many IPV victims due to a lack of established social support systems. Mazza, Maranoa, Laib, Janiria, and Sania (2020) have suggested that COVID-19 stay-at-home orders and social distancing will likely lead to a woman's home becoming one of the most dangerous places for IPV victims due in large part to the requirement to quarantine day-after-day with their violent and abusive partner with limited access to those that might provide care and assistance. The economic impact of the COVID-19 pandemic, record levels of male unemployment, added stressors in the home, including the care and home schooling of children, and the social distancing measures required by the epidemiological response, may serve to undermine the decades of progress made in keeping women and children safe at home. A recent article in the New York Times by Taub (2020) suggests that "movement restrictions aimed to stop the spread of the coronavirus may be making violence in homes more frequent, more severe, and more dangerous." The irony may be that in keeping many citizens safe from the health risks of COVID-19, we may be placing many women and children at greater risk for family violence.

\section{The Relationship between Natural Disasters and Epidemics on IPV}

In looking at the way in which the COVID-19 pandemic is likely to shape the incidence, prevalence, and impact of IPV it is important to note that scholars have explored how past natural disasters and epidemics have shaped trends in rates of violence, impacted the health and well-being of IPV victims, but also exacerbated the factors associated with violent behaviors and victimization. This includes work in the 
United States and abroad on the impact of hurricanes, earthquakes, and other natural and man-made disasters (Parkinson, 2019; Campbell \& Jones, 2016; Chan \& Zhang, 2011; Buttell \& Carne, 2009). There is strong evidence to suggest that women's physical and mental health and the risk of IPV is connected to the consequences of natural disasters and pandemics, including social isolation, economic instability, and increasing relationship and family conflict. Given the likely impact of the COVID-19 pandemic on the risk for a number of consequential health impacts, Galea, Merchant, \& Lurie (2020) point to the need for investments in efforts to ensure historically marginalized groups and those likely to be isolated during the pandemic receive outreach services. This includes older adults, women with historic victimization experiences, and those with mental illness and chronic health conditions.

Drawing on police calls for service and arrest data from the New Orleans Police Department, both before and after Hurricane Katrina, Buttell and Carney (2009) found a significant increase in calls for service, along with increased rates of arrests for domestic violence related offenses. They also found an increase in the severity of IPV in the post-hurricane data. Most importantly, Buttell and Carney (2009) note that the New Orleans Police Department actively responded to IPV calls for service following Hurricane Katrina and that there was not an agenda to prioritize other demands on police time during and after the crisis.

Bell and Folkerth (2016) have shown that survivors of natural disasters have significant health sequelae and that PTSD and depression are commonly seen in women who have lived through a natural disaster. Their work also draws on data post-Hurricane Katrina and showed that rates of violence against women rose from 4.6 cases per 100,000 per day to well over 16 cases per 100,000 per day among displaced populations following the hurricane. They found that one year after Hurricane Katrina those victims housed in FEMA trailer parks reported higher levels of negative mental health as compared to non-victims.

In contrast, work by Bell \& Folkerth (2016) note evidence suggesting that for some couples natural disasters may enhance intimate relationships, particularly among those individuals and couples with a strong sense of community and social cohesion, along with those with a strong integration into the workforce. These unprecedented events may provide increased time for couples to share coping mechanisms to manage the stress of these disasters, and may also provide motivation and incentives to draw on social support systems that enhance their intimate relationship and relationships with others.

Work by Jenkins and Phillips (2008) notes that while domestic-violence victims and survivors experienced heightened levels of violence during Hurricane Katrina and its aftermath, even in that difficult context, some women made the choice to call the police, report the violence, and leave their abusive situations. This suggests that the stress and impact of natural disasters and pandemics may impact the decision-making of IPV victims. They also noted that victim advocates responded in new ways to help these women meet their unique needs as both victims of IPV and post-Katrina survivors. As with these natural disasters, and perhaps the COVID-19 pandemic, there may be an impetus for some women with long histories of IPV to report to the police and seek assistance from diverse medical and social service entities. It may be that the pandemic will serve at the final crisis situation in their violent relationship. These natural disasters may therefore serve as a cathartic event for victims of IPV, leading to a 
turning point in attempts to bring an end to a violent relationship. For some victims, the pandemic may create the context in which they will proceed through what Burke, Gielen, McDonnell, O'Campo, \& Maman (2001) note as the key behavioral changes needed for ending and fleeing a violent relationship. This includes assessing the options for and preparing for leaving, selection of action(s), and the use of safety strategies to remain free from violence and abuse.

\section{What Do we Know So Far? Early Data on the Impact of COVID-19 on IPV}

It will be challenging to fully document the early impact of the COVID-19 pandemic on the incidence and impact of IPV due to the hidden nature of violence at the hands of an intimate partner. The low rates of police reporting and the challenges for scholars to quickly deploy a research plan that would be able to draw on diverse methodologies to measure victimization experiences among a representative sample of the population, will impact the ability to assess the connection between COVID-19 and IPV. This is a consequence of the under-reporting of these crimes to law enforcement agencies, the under-utilization of victim and social service agencies by victims, and the challenge of collecting self-report victimization data during the pandemic. Most importantly, researchers do not yet have access to the types of data that would most readily allow for an examination of the relationship between COVID-19 and IPV.

A recent article by the World Health Organization (2020) notes that many of the survey research methodologies drawn on traditionally in the violence against women literature, will not be practical during the pandemic and others research strategies may place women at risk of violence. Yet, there is some early data from the United States and abroad that suggests there have been increased reports of IPV during the pandemic, particularly increases in officially reported IPV to police agencies, emergency rooms, domestic violence hotlines, and social service agencies. It is important to note that police reported crimes and victimization are not clearly correlated to actual levels of criminal and violent behavior. Increased reporting to the police and victim service agencies may be (and is likely to be) a function of an increased number of victimization experiences of those victims who are most apt to call the police to report their experiences. These are more likely to be victims with the economic resources to be able to navigate ending a violent relationship and have the social support systems to assist. Changes in official reporting rates may also be a function of a change in the experiences and decision-making of those who were victims of IPV prior to the pandemic. The pandemic may serve as the catalyst for victims to report their experiences.

Importantly, the early data on the relationship between COVID-19 and IPV come from news agencies who report data from police agencies, domestic violence shelters, and/or emergency rooms from individual or small groups of cities. For example, Taub's (2020) New York Times article notes evidence for the relationship between COVID-19 and increased rates of IPV citing significant increased calls to domestic violence emergency support lines in China, Italy, and Spain. Yet the source, nature, and quality of the data for these reports is not clear. Bradbury-Jones and Isham (2020) similarly note that the leading IPV organization in the United Kingdom reported that calls to its domestic violence hotline increased by $25 \%$ within the first week of the tighter social distancing and stay-at-home measures. Similarly, Azpirir (2020) also notes that a 
Vancouver-based domestic violence crisis line had experienced a 300\% increase in calls amid the COVID-19 pandemic. Victims have also increased their utilization of Internet based resources. This includes increased Internet browsing traffic to a United Kingdom-based website, which has seen a $150 \%$ surge in traffic after the government lockdowns (Bradbury-Jones \& Isham, 2020). Campbell (2020) has suggested that "the growing global trend of increasing reports of domestic violence cases is likely to continue throughout the pandemic and may only represent a "tip of the iceberg" as many victims still find themselves trapped with the perpetrator and unable to report the abuse." The increasing sale of guns and ammunition during the pandemic also give rise to added concern for women's safety, given the link between firearm access and the risk of lethal IPV. An additional concern is the increased risk of children's exposure to IPV given stay-at-home orders and school cancellation.

These are alarming increases in documented reporting via law enforcement agencies and victim service providers with consequential health and mental health impacts. Importantly, they do not have the ability to tell us about the experiences of victims that do not seek assistance or are unable to make such calls due to shared quarantining with their abuser. What will be needed as we proceed through the pandemic is the triangulation of diverse data sources from both criminal justice and social service agencies and self-report victimization data to explore the relationship between COVID-19 and IPV. To fully understand the extent of IPV as function of the stressors associated with COVID-19, stay-at-home orders, and social isolation researchers will need to identify the characteristics of the women and children that are/were most at risk during the pandemic.

Research by Piquero, Riddell, Bishopp, Narvey, Reid, and Piquero (2020), published in this special issue, provides an initial exploration of the way in which the stayat-home measures associated with the COVID-19 pandemic may be correlated with domestic violence calls for service. Using domestic violence incident reports for Dallas, Texas and an intervention time-series methodology, they compared domestic violence crimes for an 83-day period before the mandated stay-at-home orders to a 35-day period thereafter. They conclude that there is some evidence pointing to a short-term increase in the two weeks immediately following the stay-at-home orders in Dallas, but also note a decrease after the initial rise in officially reported IPV incidents. The authors are cautious to attribute the increase to COVID-19 orders as the cause of this increase, as they note, appeared to have been part of an increasing trend in officially reported IPV prior to the stay-at-home orders. They also note that the short-term upward trend in domestic violence incidents may have been associated with citizens voluntarily following CDC recommendations to social distance and quarantine. Piquero, et al. (2020) also note that work looking to examine the association between COVID-19 lockdown orders and changes and trends in domestic violence need to recognize the importance of exploring the factors associated with the economic implications of the pandemic that are associated with the risk of IPV, including the social isolation associated with remote work and the financial stress of layoffs and furloughs.

\section{COVID-19 and IPV: What the Different Types of Data on IPV Will Tell us}

The longstanding body of research on the reporting (and under-reporting) of IPV to the police will be important in the context of the work that will be needed to explore the 
connection between COVID-19 and IPV. The extensive scholarship on police reporting and IPV highlights the fact that the domestic violence crimes brought to the attention of the criminal justice system are not representative of all incidents of IPV (or IPV victims), and that the majority of these crimes remain unrecorded by official statistics. Police calls for service are therefore not a good measure of true incidence of IPV, and this may be particularly true during COVID-19.

There are a number of factors that are likely to shape increases, stability, or declines in IPV calls for service to the police, and the incidence of actual violent behavior and victimization is just one of those. These are discussed below. If we rely on data on social service utilization during COVID-19, we are also likely to see a decline in this type of help-seeking and reporting because of social distancing measures that will prevent victims from accessing services. We will therefore need to expand our data collection efforts beyond police reported incidents, emergency room admissions, shelter data, and data from diverse social service providers. In examining women's risk for intimate partner violence during COVID-19, self-report victimization surveys offer an advantage over official law enforcement estimates. The use of large-scale representative victimization data will help avoid some of the problems associated with law enforcement and clinical sample bias, providing researchers with a larger estimate of the extent and dynamic nature of IPV. Yet a different form of selectivity will bias these estimates, particularly during COVID-19. Self-report data typically underrepresent the most severe and violent forms of intimate partner violence and abuse, particularly among women with chronic health conditions and disabilities. The samples from self-report data may therefore not include all women who are the victims of the most severe types of IPV or those who are victims of COVID-19 disease. This points to the need for careful joint analyses of self-report survey data, estimates from law enforcement agencies, and clinical data during and after COVID-19 to tap diverse types of intimate partner abuse and also explore the way in which COVID-19 disease progression may place women at further risk for physical violence, emotional and financial abuse, and coercive control. This may be particularly true for older victims of IPV, and would be exacerbated by COVID-19 disease and symptoms. This points to the need for leadership at the state and national level to organize and coordinate data collection for research and policy decision-making. We likely will not have a good selfreport measure of IPV during the pandemic, perhaps some researchers will be able to measure in smaller regions with National Science Foundation rapid grants and other funding mechanisms, but a national level data collection seems unlikely. If this were to happen, the leadership for the National Crime Victimization Survey would need to move very quickly to develop measures that could tap changes in IPV, both during and immediately after the pandemic, but also measure the factors that might correlate with both IPV and COVID-19, including the social isolation and stressors associated with social distancing and quarantine and the economic impacts of the pandemic on families.

\section{IPV and Police Reporting: What Could we See in Terms of Police Calls for Service and why?}

There are a number of predictions that could be made with regard to the impact of COVID-19 and police reports of IPV. It is likely that we will see an increase in domestic violence calls for service to the police because of an actual increase and 
change in the level of violent behavior by some batterers and abusers. This is likely to be the case among the same set of women who were victims of IPV prior to COVID19, and who might also during COVID-19 experience a greater number of individual incidents of violence by their previously abusive partner and perhaps more severe types of abuse. This increase in the incidence and severity of violence by historic abusers may lead to new reporting behaviors by these victims, and/or someone else in their household or neighborhood might increase their reporting to the police. It is also likely that we will see a new set of victims experience violence due to the consequences of social distancing measures and stay-at-home orders. These measures have placed those most vulnerable to violence and abuse in close proximity to their potential abuser, and this may lead to an increase in the risk factors associated with IPV. The cause of this increase is likely to be shaped by a variety of factors that are associated with IPV more generally, but that will be more prevalent during the COVID-19 pandemic. This includes social isolation and increased attempts by abusers to exert power and coercive control, unemployment, economic distress, marital conflict, and substance use and abuse. These are discussed further below. The COVID-19 epidemic may also serve at a turning point for some victims of IPV, creating the crisis and catharsis that leads to attempts to flee and end the violent relationship.

Alternatively, the rates of police reporting (victim calls for service) during COVID19 could stay relatively flat. We might actually see those who have been victims of IPV prior to the pandemic to now be less apt to call the police during COVID-19. So, while actual behaviors by offenders may stay the same or incidents of violence may go up during the pandemic, some victims during COVID-19 may be less likely to call the police or seek help. This could be for a number of reasons, including the lack of access to a secure place to call the police and reach out for help, and/or the inability to find a safe place to research options for leaving (including safety planning, organizing paperwork, and packing) due to quarantine and stay-at-home orders. The lack of economic resources to leave the abuser during the pandemic and fear of reprisal by the offender, leading to greater violence, will also impact victim decision-making.

Rates of police reporting may also decline. There may be some women who will be less likely to call the police during the pandemic because they want to reserve these essential services for those in immediate need - specifically COVID-19 patients. It may be that during COVID-19, that in some jurisdictions, rates of police reporting could go down, and rates of police reporting among some types of IPV victims decline. Actual violent behaviors may stay the same (or go up) or incidents of violence experienced by women who have been experiencing IPV prior to the pandemic may stay the same (or go up), but now women are even less likely during COVID-19 to call the police to seek help for fear of reprisal and inability to find a safe place to call due to social distancing. Illness associated with COVID-19 disease may also impact a victim's ability to reach out for assistance with IPV. Calls for service would therefore decline during this time.

\section{Stay-at-Home Is Not Safe at Home}

During COVID-19 social distancing and stay-at-home orders may expand a current perpetrator's coercive control and power over their victim. With victims and perpetrators in close proximity, batterers will be able to increase their ability to be in control of decision-making, determine day-to-day outcomes, and monitor and socially isolate 
their victim from family and friends. Bradbury-Jones and Isham (2020) note that the home is often a sphere where the dynamics of power are distorted and subverted by perpetrators. During COVID-19 this is made worse due to the lack of outside scrutiny from family and friends. For many victims, this will lead to increased physical violence, emotional and financial abuse, and coercive control. Alternatively, for those victims who have learned to adapt to their batterers control techniques and read the cues for their use of violence and abuse, the use of IPV by some batters may decline. Given stay-at-home orders, the batterer will be better able to exert day-to-day control over household decision-making, and there may be less perceived "need" by the abuser to use physical violence since the batterer is able to exert control and manipulate their victim. During stay-at-home orders, abusers may be better able to know where their victims are and control their victim's access to their family and friends - preventing access via the phone, computer, and/or other technologies. For these batterers, their ability to gain control and manipulate their victim, may lead them to not "need" to use physical methods to gain coercive control.

There are a number of elements of the COVID-19 pandemic that may also serve as mechanisms of control by abusers. For example, batterers use a variety of methods to exert control over their victims and keep their victims in a constant state of fear. These may be expanded during the pandemic. COVID-19 may be used by offenders (both those currently with their victim and those who are separated or divorced) as a way to control the victim via threats to expose them and their shared children to the novel coronavirus. Victims may also fear their abuser taking their children outside of the home during the outbreak and not be able to control who their children are exposed to or whether proper hygiene and social distancing measures are practiced. Other violent partners (and ex-partners) may use a victim's risk for COVID-19 (sucha as those who are healthcare providers, grocery store workers, working in transportation, etc.) as a way to seek custody of their shared children during the pandemic and this custody may continue after the pandemic. Healthcare providers may be particularly vulnerable to this type of abusive behavior since children may be social distancing with the abusive partner while the victim is forced to quarantine in another area of their home or outside of the home due to their ongoing risk for COVID-19.

\section{The Connection between COVID-19 and the Causes of IPV}

The risk factors for IPV victimization and the factors that limit women's ability to leave violent relationships include economic dependence on male partners (i.e., lack of education, income, and employment) and the responsibility for children both before and after divorce. A history of violent victimization also places women at risk. Demographic factors also shape risk, including age (younger), race/ethnicity, and marital status (cohabitation and dating). Finally, male partners with drug and alcohol problems, chronic unemployment, and prior intimate partner violence are at greater risk for perpetration, and these factors may be particularly prevalent during the COVID-19 pandemic. It is difficult to know whether during the pandemic we might see violence by new offenders (men who were not previously violent to their partner) or an increase in violence by previously violent men due to increased proximity and exposure between victims and offenders, and the exacerbation of these risk factors for IPV. The question researchers will need to explore both during the early stages of the 
pandemic and long-term is whether there has been (or will be) an increase in IPV among men who were not previously violent due to factors that enhance the risk for IPV that also correlate with the COVID-19 pandemic (see below). Alternatively, will we may be most likely to see an increased incidence and severity of violence by perpetrators who were previously violent, but now COVID-19 has exacerbated the role of those risk factors.

\section{IPV Risk and Protective Factors during COVID-19}

Stress frustration perspectives posit that any form of negative affect or distress is likely to increase the likelihood of violence and aggression. Stressful life events produce aggression and violence because they create negative affect. Stress-frustration theories (Holtzworth-Munroe, Bates, Smutzler, \& Sandin, 1997) suggest that diminished economic resources within the family lead to stress, frustration, and conflict in intimate relationships that heightens the risk of male-perpetrated violence against women. This suggests that during COVID-19 women with unemployed partners would be at the greatest risk for IPV and abuse. The economic uncertainty during the pandemic and record levels of unemployment are likely to add to the stress experienced by both men and women, heightening the risk of marital conflict and violence.

Economic and marital dependency of female partners on their husbands will place many women at risk for new or continued IPV during COVID-19, and also create financial challenges to women navigating ending a violent relationship. Women's lack of employment and financial resources during the pandemic, and their disproportionate responsibility for children will limit their ability to end violent relationships and/or identify mechanisms for leaving their abuser. Some Feminist research (Kaukinen, 2004; Anderson, 1997) also suggests that economic variables are often symbolic in nature and that men who do not have access to marital power via employment and economic resources will use violence in an attempt to re-establish their power at home, given their lack of access to economic resources to establish a traditional masculinity. The financial stress of the COVID-19 pandemic will be unprecedented. We have already seen the highest rates of unemployment in the last 50 years. For those couples in which the female partner may be able to continue working (remotely or otherwise), while the male partner is unemployed, will experience a shift in economic and symbolic power, particularly among couples in which the male partner previously held that "breadwinner" role.

In exploring the role of COVID-19 and the risk of IPV, social distancing and stay-athome orders will likely lead to increased conflict, disagreements, and arguments due to increased daily proximity of couples. This in turn could lead to an increased prevalence of common couple violence among both couples with and without previous IPV. Johnson (1995) notes that this type of intimate partner violence does not include severe acts of violence, is likely to be mutual between partners (gender symmetric), and does not exhibit a general pattern of coercive control by the male partner. A recent New York Times article by Taub (2020) on China noted that some victims stated that "during the epidemic, we were unable to go outside, and our conflicts just grew bigger and bigger and more and more frequent," "everything was exposed." For some couples, particularly those experiencing financial and family stressors during the pandemic, they will likely have an increase in the number of arguments and conflicts during sustained social isolation and physical proximity. Increases in common couple violence is a likely 
consequence of the pandemic, particularly among young and newly formed intimate relationships.

The risk and impact of IPV during the COVID-19 pandemic may also be shaped by rates of drug and alcohol use and abuse. There is research (Brooks, Webster, Smith, Woodland, Wessely, Greenberg, \& Rubin, 2020) to suggest that quarantine and other social isolating conditions are associated with alcohol abuse, depression, and posttraumatic stress symptoms. While researchers (Fals-Stewart, 2003; Leonard \& Quigley, 1999) have pointed to a strong correlation between drinking, and in particular heavy alcohol consumption, and the risk of intimate partner violence, the exact mechanism is less clear. Some scholars have suggested that problematic drinking and the risk of IPV perpetration may share a common etiology, a need to achieve personal power and control. My own research has shown that women with male partners who engage in heavy episodic drinking are also more likely to engage in both physical violence and coercive control. The exact relationship between alcohol (and drug) use and abuse and the risk of IPV is complicated. Substance abuse, along with the economic stressors associated with COVID-10 will provide perpetrators with an excuse (and justification) for their violent and abusive battering behaviors, and an explanation for victims to excuse their abuser. Alcohol allows perpetrators to justify their violent behavior, and for victims to explain it and excuse it. It is therefore best to view substance use as a risk marker for IPV, while not a direct cause. At the same time, drinking may increase the frequency or severity of male perpetrated violence against women. Clay and Parker (2020) note the importance of public health approaches that explore the public health effects of long-term social isolation related to COVID-19 on alcohol use and misuse noting the need to protect the most vulnerable individuals from excessive alcohol consumption during the pandemic. In addressing the connection between substance use and the stressors associated with COVID-19, Da, Im, \& Schiano (2020) note the need to put in place awareness and telehealth strategies to curb what is likely to become a serious consequence of the coronavirus pandemic.

\section{Summary: Implications and where Should we Place our Resources}

Many IPV victims will be reluctant to seek help from healthcare providers or engage in social service utilization during the pandemic. For some victims, they may be reluctant to seek healthcare or other emergency care for their IPV-related injuries due to the fear of contracting COVID-19 due to enhanced exposure in healthcare settings. Other victims might believe they should reserve those healthcare services for the people most in need, those suffering from COVID-19 disease. Many IPV victims will have reduced access to victim services during the pandemic since many of these services are limited by social distancing and stay-at-home orders and are only operating remotely. Remote type therapies and services are likely to place many victims at risk for further violence by their abuser or may lack access to phones and internet during COVID-19 due to financial hardships or coercive control by their abuser. Access to housing and other advocacy services will continue to be limited during the early days of stay-at-home orders due to social distancing and quarantine requirements, with many of these services temporarily shut down, including courthouses for example. A fall outbreak of the pandemic will make worse women's experiences with IPV and further limit their options for leaving a violent partner. 
There will be the need for strong federal and state leadership to expand victims' access to support services and economic resources during the pandemic. We will need to be creative in our thinking on how to outreach to victims and provide awareness messaging to bystanders. We will need to create ways in which IPV victims will be able to safely access both in-person and telehealth options in discrete ways without alerting their perpetrator. Bradbury-Jones and Isham (2020) also point to the importance of protecting the advocates who work in a voluntary manner to support victims and the need for personal protective equipment to be provided. Boserup, McKenney, \& Elkbul (2020) note the importance of making IPV screening tools and assessments more readily available in diverse clinical settings and among telehealth providers during the pandemic. Social workers, advocates, and others who work with victims will need to identify ways to safely work with victims on safety planning and self-care, but also plans for victims to leave their violent partner. There will need to be collaboration and creative thinking on how to expand the availability of services and diversify the nature of transitional housing, particularly for victims who may have contracted or been exposed to COVID-19. For those victims who report to the police, there will be a need for more intensive police and social service follow-up both during and after the COVID-19 pandemic. This will be particularly true for women who remain in their home with the abuser. Finally, we will need to change the way in which awareness campaigns have provided information to IPV victims and bystanders. Reliance on public spaces for this information is no longer the most effective way to outreach to victims. Boserup, McKenney, \& Elkbul (2020) therefore suggest that diverse social media outlets should seek to identify ways to reach a wider audience during city lockdowns and stay-at-home orders.

\section{References}

Anderson, K. L. (1997). Gender, status, and domestic violence: An integration of feminist and family violence approaches. Journal of Marriage and the Family, 59, 655-669.

Bell, S. A., \& Folkerth, L. A. (2016). Women's mental health and intimate partner violence following natural disaster: A scoping review. Prehospital Disaster Medicine, 31(6), 648-657.

Boserup, B., McKenney, M., \& Elkbuli, A. (2020). Alarming trends in US domestic violence during the COVID-19 pandemic. The American Journal of Emergency Medicine. https://doi.org/10.1016/j. ajem.2020.04.077.

Bradbury-Jones, C., \& Isham, L. (2020). The pandemic paradox: The consequences of COVID-19 on domestic violence. Journal of Clinical Nursing. https://doi.org/10.1111/jocn.15296.

Brooks, S. K., Webster, R. K., Smith, L. E., Woodland, L., Wessely, S., Greenberg, N., \& Rubin, G. J. (2020). The psychological impact of quarantine and how to reduce it: Rapid review of the evidence. The Lancet, 395(10227), 912-920.

Buttell, F. P., \& Carney, M. M. (2009). Examining the impact of hurricane Katrina on police responses to domestic violence. Traumatology, 15(2), 6-9.

Burke, J. G., Gielen, A. C., McDonnell, K. A., O'Campo, P., \& Maman, S. (2001). The process of ending abuse in intimate relationships: A qualitative exploration of the transtheoretical model. Violence Against Women, 7(10), 1144-1163.

Campbell, A. M. (2020). An increasing risk of family violence during the Covid-19 pandemic: Strengthening community collaborations to save lives. Forensic Science International: Reports, 2(100089).

Campbell, L. M., \& Jones, S. J. (2016). An innovative response to family violence after the Canterbury earthquake events: Canterbury family violence collaboration's achievements, successes, and challenges. Australasian Journal of Disaster and Trauma Studies, 20, 89-100. 
Catalano, S. M. (2012). Intimate partner violence, 1993-2010. Washington, DC: US Department of Justice, Office of Justice Programs, Bureau of Justice Statistics.

Chan, K. L., \& Zhang, Y. (2011). Female victimization and intimate partner violence after the may 12, 2008 Sichuan earthquake. Violence and Victims, 26(3), 364-376.

Clay, J. M., \& Parker, M. O. (2020). Alcohol use and misuse during the COVID-19 pandemic: A potential public health crisis? The Lancet: Public Health.

Da, B. L., Im, G. Y., \& Schiano, T. D. (2020). COVID-19 hangover: A rising tide of alcohol use disorder and alcohol-associated liver disease. Hepatology. https://doi.org/10.1002/hep.31307.

Fals-Stewart, W. (2003). The occurrence of partner physical aggression on days of alcohol consumption: A longitudinal diary study. Journal of Consulting and Clinical Psychology, 71(1), 41-52.

Galea, S., Merchant, R. M., \& Lurie, N. (2020). The Mental Health Consequences of COVID-19 and Physical Distancing. In The mental health consequences of COVID-19 and physical distancing: The need for prevention and early intervention. JAMA Internal Medicine.

Holtzworth-Munroe, A., Bates, L., Smutzler, N., \& Sandin, E. (1997). A brief review of the. research on husband violence. Part I: Maritally violent versus non-violent men. Aggression and Violent Behavior, 2 , 65-99.

Jenkins, Pam, and Brenda Phillips. (2008). Battered women, catastrophe, and the context of safety after Hurricane Katrina." NWSA Journal 20(3), 49-68.

Kaukinen, C. (2004). Status Compatibility, Physical Violence, and Emotional Abuse in Intimate Relationships. Journal of Marriage and Family, 66(2), 452-471.

Lauritsen, J. L., \& Heimer, K. (2009). Gender and violent victimization, 1973-2005. Washington, DC: National Institute of Justice.

Leonard, K. E., \& Quigley, B. M. (1999). Drinking and marital aggression in newlyweds: an event-based analysis of drinking and the occurrence of husband marital aggression. Journal of studies on alcohol, 60(4), 537-545.

Mazza, M., Marano, G., Lai, C., Janiri, L., \& Sani, G. (2020). Danger In Danger: Interpersonal Violence During COVID-19 Quarantine. Psychiatry Research, 113, 046.

Parkinson, D. (2019). Investigating the increase in domestic violence post-disaster: An Australian Case Study. Journal of Interpersonal Violence, 34(11), 2333-2362.

Piquero, A. R., Riddell, J. R., Bishopp, S. A., Narvey, C., Reid, J. A., \& Piquero, N. L. (2020). Staying Home, Staying Safe? A short-term analysis of COVID19 on Dallas domestic violence. American Journal of Criminal Justice.

Powers, R. A., \& Kaukinen, C. E. (2012). Trends in intimate partner violence: 1980-2008. Journal of Interpersonal Violence, 27(15), 3072-3090.

Taub, A. (2020, April 6). A New Covid-19 Crisis: Domestic Abuse Rises Worldwide. New York Times. Retrieved from https://www.nytimes.com/2020/04/06/world/coronavirus-domestic-violence.html.

World Health Organization. (2020, April 17). Violence Against Women and Girls Data Collection during COVID-19. Retrieved from https://www.unwomen.org/-/media/headquarters/attachments/sections/library/publications/2020/vawg-data-collection-during-covid-19-compressed.pdf?la=en\&vs=2339.

Xie, M., Heimer, K., \& Lauritsen, J. L. (2012). Violence against women in US metropolitan areas: Changes in women's status and risk, 1980-2004. Criminology, 50(1), 105-143.

Publisher's Note Springer Nature remains neutral with regard to jurisdictional claims in published maps and institutional affiliations.

Catherine (Katie) Kaukinen Ph.D., is a professor and chair in the Department of Criminal Justice at the University of Central Florida. Her interests include intimate partner violence, developmental antecedents and long-term consequences of victimization, victim coping, resilience, help-seeking, and decision-making, the history of Title IX and Federal initiatives to address violence against college women, and the evaluation of campus-based violence against women prevention and intervention programs. Her work on developing programs on campus to address violence against women includes over $\$ 1$ million dollars in funding from the Office on Violence Against Women in which she developed a multi-campus victim service intervention and prevention program addressing dating violence, intimate partner violence, sexual assault, and stalking. Her research has appeared in Criminology, Journal of Marriage and Family, Journal of Research in Crime \& Delinquency, Journal of Interpersonal Violence, Trauma, Violence, and Abuse, and Violence \& Victims, among other outlets. 